\title{
Infraestrutura para um Corpo de Conhecimento em Melhoria de Processos de Software Baseado no MR-MPS-SW
}

\author{
Peter P. Lupo ${ }^{1}$, Marcos Kalinowski ${ }^{2}$, Ana Regina C. da Rocha ${ }^{1}$ \\ ${ }^{1}$ COPPE/UFRJ - Universidade Federal do Rio de Janeiro - Caixa Postal 68511 - \\ CEP 21.941-972 - Rio de Janeiro, RJ - Brazil \\ ${ }^{2}$ Instituto de Computação - Universidade Federal Fluminense (UFF) \\ Rua Passos da Pátria 156, 24.220-240 - Niterói - RJ - Brazil \\ \{pplupo, darocha\}@cos.ufrj.br, kalinowski@ic.uff.br
}

\begin{abstract}
Software development organizations commonly follow maturity models to guide their software process improvement initiatives, being responsible for identifying and choosing the technologies to address these models' requirements. This paper proposes an infrastructure to organize a body of knowledge of technologies that support the implementation of the MR-MPS$S W$ focused on the software process improvement professionals' needs.
\end{abstract}

Resumo. Organizações desenvolvedoras de software comumente fazem uso de modelos de maturidade para orientar a melhoria de seus processos, sendo responsabilidade destas organizações identificar e escolher as tecnologias para apoiar o atendimento dos requisitos destes modelos. Este trabalho propõe uma infraestrutura para organizar um corpo de conhecimento de tecnologias que apoiam a implementação do MR-MPS-SW voltado para as necessidades dos profissionais de melhoria de processos.

\section{Introdução}

Diferentes fatores motivam as organizações a realizarem melhorias em seus processos de desenvolvimento de software. Muitas vezes estas melhorias são realizadas com o apoio de modelos de maturidade como o CMMI-DEV [CMMI Product Team 2010] e o MR-MPS-SW (Modelo de Referência MPS para Software) [Softex 2016]. Modelos de maturidade são comumente descritos na forma de objetivos ou resultados esperados com a execução dos processos da organização. Estes resultados são decorrência da utilização de boas práticas. A adoção destas boas práticas, por sua vez, pode fazer uso de diferentes tecnologias (métodos, técnicas e ferramentas) [CMMI Product Team 2010; Softex 2016].

Dentre os modelos de maturidade de processos de Engenharia de Software, o MR-MPS-SW se destaca no âmbito nacional, apresentando inclusive maior adoção que o CMMI-DEV [Kalinowski et al. 2014]. Também é interessante ressaltar que a adoção do MR-MPS-SW pela indústria tem sido relacionada com benefícios para as organizações como o crescimento organizacional, aumento da quantidade de projetos, aumento da quantidade de clientes e aumento da quantidade de funcionários contratados [Travassos and Kalinowski 2014].

Nas organizações que utilizam o MR-MPS-SW, a busca e seleção de tecnologias que apoiem as melhores práticas para o atendimento dos requisitos do modelo são res- 
ponsabilidade de cada organização. O modelo indica "o que deve ser feito", mas não "como deve ser feito". Esta seleção deve, portanto, ser feita de maneira compatível com o contexto, os objetivos estratégicos da organização e a cultura organizacional, o que exige conhecimento tanto nos processos a serem melhorados quanto nas tecnologias de Engenharia de Software [Cerdeiral 2014; Kalinowski et al. 2010; Santos et al. 2012].

A busca por conhecimento a respeito de tecnologias de Engenharia de Software que apoiem a melhoria de processos é essencial podendo levar as organizações a realizarem treinamentos, contratarem novos colaboradores, consultorias, participarem de congressos ou recorrerem à literatura para alcançar seus objetivos e maximizar seu retorno de investimento [Cerdeiral 2014; Kalinowski et al. 2010; Montoni 2010]. É possível encontrar na literatura estudos investigando tecnologias, com resultados de pesquisas da academia e de aplicações na indústria. Este conhecimento está disponível em diferentes bases científicas, sendo algumas de acesso restrito. Tem-se, portanto, conhecimento gerado, mas ele se encontra disperso e nem sempre é de fácil acesso.

De acordo com KUHRMANN et al. [Kuhrmann et al. 2015], apesar de haver extenso conhecimento sobre melhoria de processos de software, é difícil responder a questões como "O que existe?", e "Qual o estado atual da área e pesquisas relacionadas?". Além disto, os conhecimentos sobre tecnologias relatados na literatura dificilmente alcançam os profissionais que atuam na indústria, sendo necessário adaptar o formato de apresentação das informações para melhor apoiar tomadores de decisão [Jedlitschka et al. 2014].

Uma alternativa para sintetizar conhecimento sobre tecnologias que apoiam a melhoria de processos e apresentá-lo de maneira organizada e estruturada é a organização de um corpo de conhecimento (BoK - Body of Knowledge), que poderia auxiliar profissionais facilitando o acesso ao conhecimento e ajudando a promover sua utilização efetiva. Um BoK é um conjunto estruturado de conhecimentos em um domínio de interesse que sejam aceitos, acordados e utilizados por profissionais que atuam neste domínio [Chinn and Kramer 1995; Oliver 2012; Ören 2005].

Este artigo descreve uma infraestrutura que permite a organização de um BoK sobre tecnologias que podem ser utilizadas na melhoria de processos de software com base no MR-MPS-SW, o MPS-BoK. O propósito do MPS-BoK é permitir organizar conhecimento sobre tecnologias (utilizadas na indústria e/ou avaliadas através de estudos experimentais) e disponibilizá-lo de maneira que possa ser utilizado por profissionais que realizam melhoria de processos com base no MR-MPS-SW. A infraestrutura foi projetada para permitir a organização do MPS-BoK de maneira incremental e colaborativa através de um processo para atualizá-lo, o MPS-BoK-P, e de uma ferramenta que automatiza a organização do conhecimento extraído neste processo e o disponibiliza no MPS-BoK, oferecendo uma possível vantagem aos tradicionais guias de implementação que normalmente apenas são atualizados juntamente com as atualizações do modelo. Os achados das avaliações realizadas fornecem indícios de sua viabilidade de utilização e subsídios relevantes para sua melhoria.

O restante deste artigo está organizado da seguinte forma. A Seção 2 descreve a fundamentação teórica a respeito de corpos de conhecimento. A Seção 3 apresenta os requisitos para a infraestrutura. A Seção 4 descreve a infraestrutura em si. As avaliações realizadas são detalhadas na Seção 5. Por fim, a Seção 6 apresenta as considerações finais do artigo. 


\section{Corpos de Conhecimento e sua Organização}

$\mathrm{Na}$ Engenharia de Software pesquisadores constantemente desenvolvem novas tecnologias, realizam experimentos e publicam resultados. É importante que o conhecimento sobre estas tecnologias seja organizado para que seja difundido e utilizado na prática. Uma forma de se realizar esta organização e estruturação de conhecimento que possa permitir uma generalização é a organização de corpos de conhecimento (Body of Knowledge-BoK) [Shull et al. 2003].

Um BoK pode ser definido como um conjunto estruturado de conhecimentos em um domínio de interesse que sejam aceitos, acordados e utilizados por profissionais que atuam neste domínio [Chinn and Kramer 1995; Ören 2005]. A percepção da importância da criação de corpos de conhecimento em uma área relativamente recente como a Engenharia de Software está representada na construção de diversos corpos de conhecimento. Em uma revisão informal da literatura foi possível identificar 15 corpos de conhecimento na área de Engenharia de Software e áreas relacionadas, como gerência de projetos e engenharia de sistemas (e.g., SWEBOK, SWE-BoK, PMBoK, SEBoK, PSP BoK, TSP BoK) e analisados em detalhes [Lupo 2016].

Uma processo para a organização de BoKs a partir de informações provenientes de publicações científicas que pode ser aplicado de maneira colaborativa é o SKE (Systematic Knowledge Engineering) [Biffl et al. 2014a; Biffl et al. 2014b]. SKE é um processo sistemático de engenharia de conhecimento definido com base nas diretrizes para revisões sistemáticas da literatura [Kitchenham and Charters 2007]. SKE apoia a organização de corpos de conhecimento a partir de estudos empíricos de publicações científicas e já foi utilizado para gerar corpos de conhecimento com evidências de estudos empíricos em inspeção [Biffl et al. 2014a] e em outras áreas, como ameaças à validade e ações de controle em experimentos controlados e linhas de produtos de software [Biffl et al. 2014b]. A Figura 1 apresenta uma visão geral do processo SKE.

Uma descrição detalhada do processo pode ser encontrada em [Biffl et al. 2014b]. A descrição resumida de suas fases visando fornecer a fundamentação para a compreensão do trabalho descrito neste artigo é apresentada a seguir.

Na primeira fase do SKE, um protocolo é construído baseado em uma configuração específica da estratégia PICO (Population, Intervention, Comparison, Outcome) [Richardson et al. 1995] para derivar questões de pesquisa que possam ser aplicadas a bibliotecas digitais no formato "P and I and $\mathrm{C}$ and O". A segunda fase do SKE compreende a criação de um modelo de domínio para a base de conhecimento. A terceira fase do SKE compreende a extração de informações dos trabalhos encontrados através de buscas realizadas utilizando o protocolo criado na primeira fase e a quarta fase compreende a integração destas informações à base de conhecimento criada na segunda fase. Também está na quarta fase a criação de um mecanismo de consulta a esta base de conhecimento.

\section{Requisitos para a Infraestrutura para a Organização do MPS-BoK}

A partir do conhecimento obtido na revisão da literatura e da experiência dos autores em melhoria de processos de software, foram definidos requisitos para o MPS-BoK, buscando o atendimento das necessidades dos profissionais que realizam melhoria de processos de software com base no MR-MPS-SW. Destes requisitos foram então derivados requisitos para a infraestrutura que apoia a organização do MPS-BoK. Um detalhamento destes requisitos segue. 


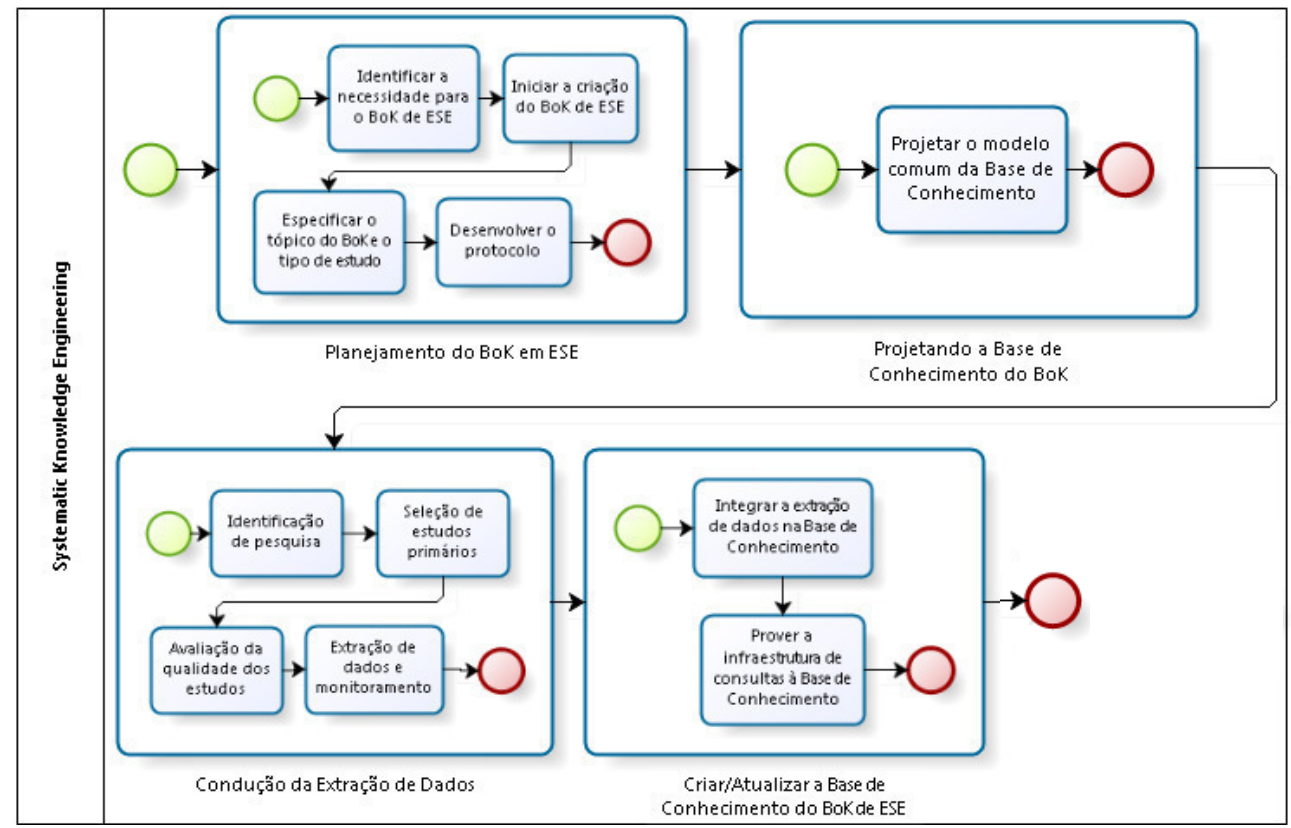

Figura 1 - Processo SKE, adaptado de Biffl et al. [2014b].

\subsection{Organizar conhecimento sobre tecnologias de acordo com a estrutura do MR- MPS-SW}

Sendo o público-alvo do MPS-BoK composto por profissionais interessados na melhoria de processos com base no MR-MPS-SW, é de se supor que conheçam os resultados esperados exigidos pelo modelo. Assim, recuperar os conhecimentos do BoK relacionados diretamente aos resultados esperados é um mecanismo simples que utiliza um vocabulário conhecido pelos usuários, sem a necessidade da organização de consultas mais complexas.

Como o MR-MPS-SW [Softex 2016] está organizado em processos de Engenharia de Software, o MPS-BoK deve ser organizado segundo os mesmos processos de Engenharia de Software encontrados no modelo. Para isto, o MR-MPS-SW foi analisado quanto à sua estrutura, que pode ser vista na Figura 2.

Cada nível do MR-MPS-SW agrupa processos com objetivos e escopo claramente definidos através de um propósito e resultados esperados. Além dos processos, cada nível possui atributos de processo (AP), que são acrescentados conforme os níveis de maturidade ascendem, e determinam a capacidade com a qual cada processo é executado.

\subsection{Disponibilizar conhecimento sobre as tecnologias de forma a apoiar os profissi- onais na tomada de decisão}

O corpo de conhecimento deve poder ser utilizado como referência pelos profissionais para tomada de decisão sobre tecnologias a serem adotadas em iniciativas de melhoria de processos. Desta forma, é importante que o MPS-BoK seja capaz de capturar conhecimento que apoie a tomada de decisão. Este conhecimento envolve informações sobre a tecnologia, o contexto em que ela pode ser utilizada e o seu impacto [Jedlitschka et al. 2014]. Neste sentido, um conjunto de informações sobre tecnologias para satisfazer as 
necessidades de informação de profissionais foi identificado [Jedlitschka et al. 2014]. A infraestrutura deve ser capaz de armazenar conhecimento que contenha estas informações sobre as tecnologias.

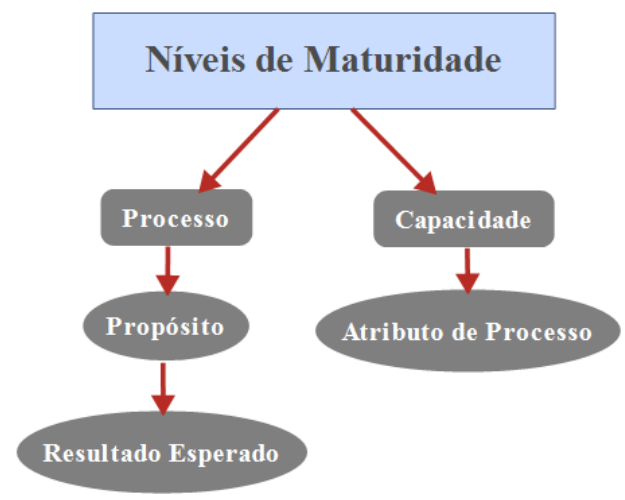

Figura 2 - Estrutura do MR-MPS-SW. Adaptado de [Softex 2016].

Atualmente existe muito conhecimento sobre tecnologias em melhoria de processos de software descrito na literatura sob a forma de relatos de experiência, relatórios técnicos, artigos, teses, etc. É bastante relevante que o conhecimento sobre estas tecnologias, desde que tenham sido aplicadas e avaliadas, possa ser utilizado para compor o MPS-BoK. Assim, é importante que a infraestrutura proposta não faça restrições quanto ao tipo de evidência ou de publicação da qual o conhecimento será extraído desde que suas contribuições sejam referentes a modelos, teorias, frameworks, guias e ferramentas, de acordo com a classificação de Kuhrmann et al. [Kuhrmann et al. 2015].

A principal forma de disponibilização deste conhecimento deve ser através da navegação pela estrutura do MR-MPS-SW. Além deste mecanismo, para facilitar a sua utilização, a infraestrutura deve fornecer um mecanismo de busca por palavras chave em cima das informações (tecnologia, contexto e impacto) contidas no MPS-BoK, permitindo a rápida recuperação do conhecimento desejado.

\subsection{Fornecer apoio para a atualização do conhecimento}

A infraestrutura deve prover uma forma que permita a atualização do conhecimento de maneira colaborativa e incremental, de forma que vários pesquisadores possam contribuir integrando novos conhecimentos ao MPS-BoK.

\section{A Infraestrutura para o MPS-BoK}

A infraestrutura foi definida objetivando o atendimento dos requisitos e é composta por dois componentes principais. O processo MPS-BOK-P, que visa permitir a organização do MPS-BoK de maneira incremental e uma ferramenta que apoia a organização do conhecimento extraído neste processo e o disponibiliza no MPS-BoK.

\subsection{Definição do MPS-BoK-P}

O MPS-BoK-P foi definido através de uma especialização do SKE realizada a partir da análise de como suas atividades poderiam ser preparadas e otimizadas para o caso específico de elaborar o MPS-BoK que pode ser vista com detalhes em Lupo [2016]. Esta 
análise foi realizada em todas as fases do SKE, consistindo na execução integral ou parcial de algumas de suas atividades visando a sua otimização.

Durante esta execução do processo, conforme previsto em SKE, foi concebido um modelo de domínio. Para o caso específico do MPS-BoK o modelo contempla a estrutura do MR-MPS-SW e permite vincular a ela informações sobre tecnologias que apoiam profissionais na tomada de decisão, de acordo com o modelo de informações definido por [Jedlitschka et al. 2014] (e.g., tecnologia, contexto e impacto). O modelo resultante pode ser visto na Figura 3. É importante ressaltar que, apesar de ter sido construído com foco no MR-MPS-SW, este modelo é compatível com todos os modelos da família MPS e modelos com estrutura similar como o CMMI-DEV e outros. Neste modelo, além da estrutura fixa do MR-MPS-SW a entidade Tag permite associar palavraschave relacionadas com os elementos do MR-MPS-SW para facilitar buscas futuras. Tecnologias de relacionadas a um resultado são retornadas quando uma palavra chave associada a este resultado é utilizada na busca. As palavras chave foram identificadas a partir da leitura do modelo por um dos autores e associadas a atributos de processo após a revisão por outro autor. Este modelo representa o conhecimento que será agregado ao MPS-BoK através da execução do processo MPS-BoK-P.

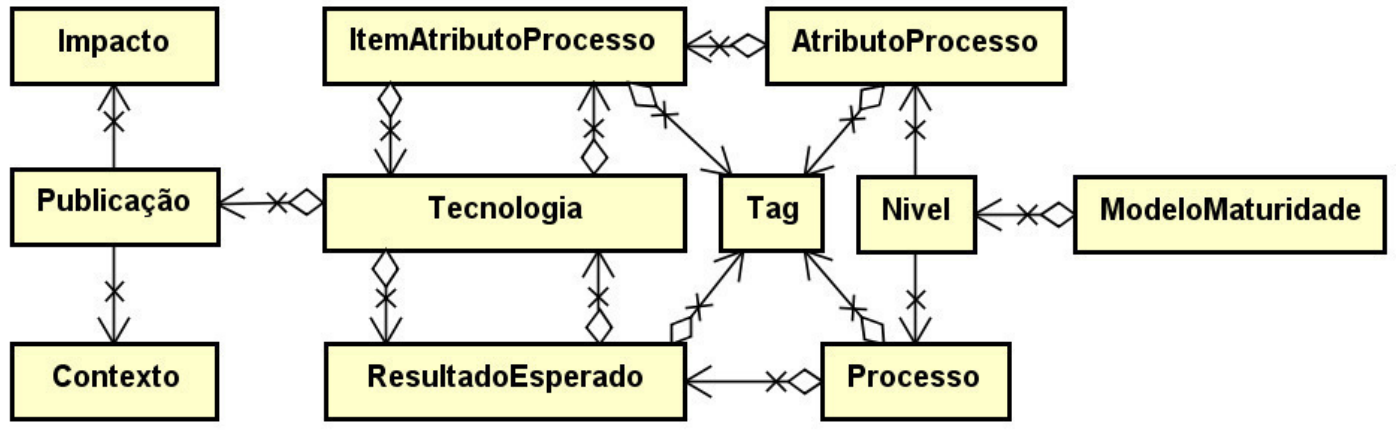

Figura 3 - Modelo de Domínio do MPS-BoK.

Apenas aquelas atividades cujos resultados variam para cada atualização do MPS-BoK estão presentes no MPS-BoK-P. As atividades do MPS-BoK-P podem ser vistas na Figura 4 e suas descrições se encontram a seguir.

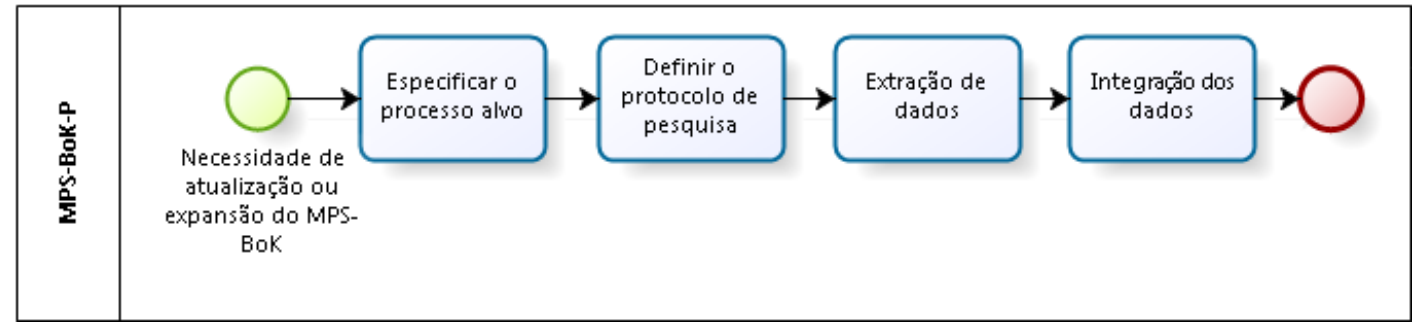

Figura 4 - Atividades do MPS-BoK-P.

Atividade 1 - Especificar o processo alvo. A primeira atividade do MPS-BoK-P é selecionar o processo para o qual conhecimento deve ser incorporado ou atualizado no BoK dentre os processos definidos no MR-MPS-SW. A partir desta seleção, pode ser definido o protocolo de pesquisa.

Atividade 2 - Definir o protocolo de pesquisa e as fontes de publicações. A atividade de definição do protocolo de pesquisa deve ser feita com base em uma configura- 
ção específica da estratégia PICO para derivar strings de pesquisa que possam ser aplicadas nas fontes de publicações.

Nesta configuração, a população representa o processo selecionado na primeira atividade. A intervenção representa o alvo da investigação (apoios na forma de técnicas, processos, métodos, metodologias, frameworks, componentes de processos ou ferramentas). Como o MPS-BoK não se restringe a uma comparação específica entre tópicos, a comparação é vazia e não será incluída na estratégia PICO. O resultado, apesar de representar elementos de interesse obrigatórios a serem extraídos dos estudos (por ex., algum tipo de melhoria de eficiência), não precisa ser definido, pois qualquer resultado é de interesse para o MPS-BoK.

Em seguida, a consulta deve ser construída no formato "(P) AND (I)". Este protocolo deve ser aplicado em uma ou mais fontes de publicações selecionadas, como bibliotecas digitais, por exemplo. Adicionalmente, a estratégia de busca pode ser complementada com estratégias de busca de publicações adicionais a partir das já selecionadas, como backward e forward snowballing.

Três critérios de inclusão obrigatórios devem ser respeitados:

1. Incluir apenas publicações relacionadas com o processo escolhido na primeira atividade.

2. Incluir apenas publicações que relatem alguma utilização ou avaliação sobre a tecnologia apresentada (e.g., experimento, survey). As publicações a serem incluídas devem ser referentes a pesquisas do tipo (de acordo com KUHRMANN et al. [Kuhrmann et al. 2015]):

- Pesquisa de avaliação - implementada na prática, avaliação da implementação conduzida.

- Proposta de solução - proposta de solução para um problema incluindo demonstração de benefícios e/ou aplicação, por exemplo, através de experimentos, estudo de caso ou avaliação com estudantes em laboratório.

3. Incluir apenas publicações cujas contribuições sejam sobre (de acordo com KUHRMANN et al. [Kuhrmann et al. 2015]):

- Modelo - representação de realidade observada por conceitos.

- Teoria - constructo de relacionamento causa-efeito.

- Framework - frameworks/métodos relacionados à melhoria de processos de software.

- Ferramenta - uma ferramenta para apoiar melhoria de processos de software

Atividade 3 - Extração de dados. A atividade de extração de dados segue as estratégias de pesquisa, seleção, avaliação e extração de dados do protocolo. Para apoiar esta atividade uma planilha de extração foi preparada para acumular dados de acordo com um modelo de conhecimento definido, que caracteriza a tecnologia em relação à estrutura do modelo MPS-SW e informações relevantes de acordo com o modelo de informações definido por JEDLITSCHKA et al. [Jedlitschka et al. 2014]. Esta planilha deve ser preenchida nesta fase com base nas informações disponíveis nas publicações selecionadas.

Atividade 4 - Integração dos dados. A integração dos dados é realizada a partir da submissão da planilha preenchida resultante da Atividade 3 para a importação e agregação ao MPS-BoK. A funcionalidade de importação na base de conhecimento é provida pela ferramenta de apoio ao MPS-BoK, detalhada na próxima seção. A partir da importação os dados ficarão disponíveis para consulta no MPS-BoK. 


\subsection{Ferramenta de Apoio ao MPS-BoK}

O acesso ao MPS-BoK é operacionalizado por uma ferramenta de apoio online que permite que seu conteúdo seja consultado e que novos conhecimentos sobre tecnologias sejam incorporados ao corpo já existente. Neste artigo são apresentadas informações gerais sobre a ferramenta, descrita em detalhes por Lupo [2016].

A ferramenta de apoio ao MPS-BoK possui dois mecanismos a partir dos quais se pode efetuar buscas por conhecimento. Um destes mecanismos é uma Full Text Search (pesquisa em texto integral), através da qual uma string de consulta pode ser informada e a ferramenta busca os conhecimentos associados. Para aumentar a flexibilidade da busca, o MR-MPS-SW foi lido e palavras-chave relacionadas ao contexto de cada resultado esperado ou atributo de processo foram identificadas. Em seguida, essas palavras chave foram associadas a todos os resultados e atributos em que apareceram. Essa informação adicional marca os resultados e atributos como etiquetas e são utilizadas nas buscas realizadas por este mecanismo.

Este mecanismo de busca pode ser visto na Figura 5, cujas características de cor originais foram alteradas para melhorar a visibilidade. Esta figura apresenta, na sua parte superior, uma caixa de texto que permite que o conhecimento seja buscado. Ao inserir uma string de consulta, o MPS-BoK apresenta na parte inferior uma tabela com o resultado da busca realizada.

\begin{tabular}{|c|c|c|c|c|c|}
\hline 20 & $\begin{array}{l}\text { MPS-Bo } \\
\text { Melhoria de Processos de Software }\end{array}$ & Kody of Knowledge & & & \begin{tabular}{|l|l|l|l|l} 
Português & \\
\end{tabular} \\
\hline \multicolumn{2}{|l|}{ Pesquisar: name1 OR name2 } & a 0 & Selecione o resultado esperado: & MR-MPS-SW & , \\
\hline \multicolumn{6}{|c|}{ Consulta realizada: name1 OR name2 } \\
\hline \multicolumn{6}{|l|}{ Resultados encontrados: } \\
\hline \multicolumn{6}{|c|}{ 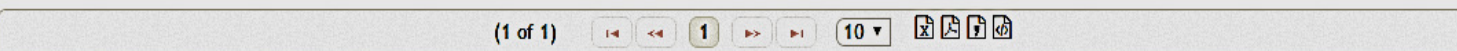 } \\
\hline Nome $\hat{v}$ & Descrição & \multicolumn{2}{|c|}{ Tipo $\hat{v}$} & \multicolumn{2}{|c|}{ Detalhes } \\
\hline Name1 & Short description1 & \multicolumn{2}{|l|}{ Ferramenta } & \multicolumn{2}{|c|}{ - } \\
\hline \multirow[t]{2}{*}{ Name2 } & Short description2 & Ferramenta & & \multicolumn{2}{|c|}{0} \\
\hline & $(1$ of 1$)$ is 44 & $\Rightarrow M \quad 10 V$ & 因团图 & & \\
\hline \multicolumn{6}{|l|}{ Como contribuir: } \\
\hline \multicolumn{6}{|c|}{$\begin{array}{l}\text { Caso você deseje contribuir conn conhecimento para este corpo de conhecimento, favor baixar o arquivo abaixo contendo un processo a ser seguido baseado en revisöes sistenátic } \\
\text { da literatura, unm modelo de planilha excel e um e-mail de contato para onde solicitar a inclusão do conhecimento identificado. }\end{array}$} \\
\hline \multicolumn{6}{|l|}{+ Download } \\
\hline \multicolumn{3}{|c|}{ @ 2016 PESC/COPPE - Programa de Engenharia de Sistemas e Computação } & \multicolumn{3}{|c|}{ UFRJ - Universidade Federal do Rio de Janeiro } \\
\hline
\end{tabular}

Figura 5 - Tela de consulta do MPS-BoK.

Outro mecanismo de busca presente na ferramenta é através da estrutura do MRMPS-SW. Assim, na ferramenta é possível navegar por níveis, processos e resultados esperados ou atributos de processo. Ao selecionar um resultado esperado ou atributo de processo, os conhecimentos associados ao elemento selecionado são retornados pela ferramenta. Na Figura 6 é possível ver os resultados esperados do processo Gerência de Projetos no nível G. Também é possível ver os outros processos e atributos de processos introduzidos neste nível.

Informações adicionais sobre a tecnologia são referentes ao contexto de utilização e ao impacto da utilização. Estas informações são específicas da utilização relatada na publicação e, portanto, estão fortemente associadas a ela. Para representar esta asso- 
ciação, estas informações são apresentadas como detalhes da publicação. Desta forma, uma mesma tecnologia pode ser identificada em mais de uma publicação e cada publicação pode trazer informações diferentes sobre contexto e impacto da utilização da tecnologia. As Figuras 7 e 8 apresentam, respectivamente, os detalhes da tecnologia e os detalhes da publicação (contexto e ao impacto de utilização da tecnologia).

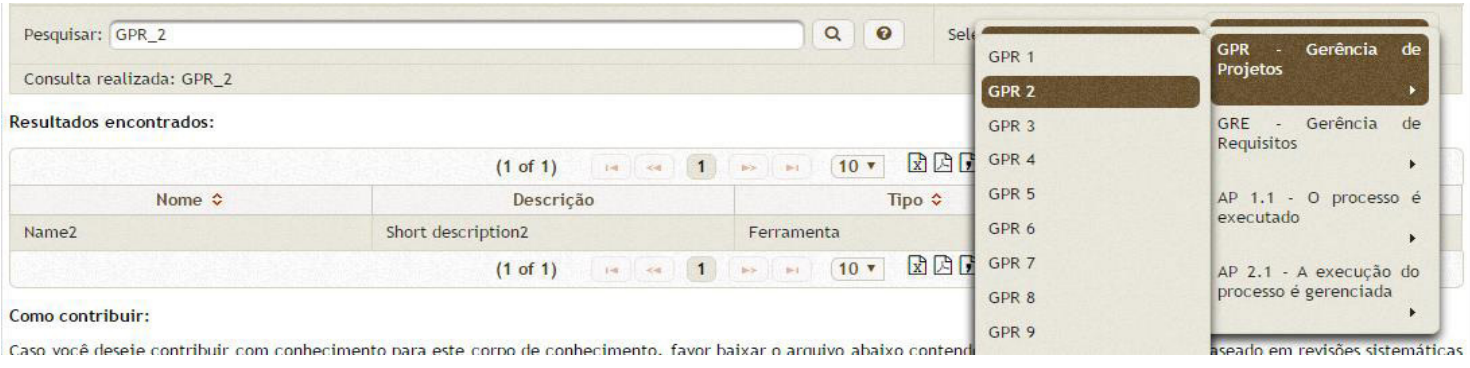

Figura 6 - Tela de consulta do MPS-BoK - detalhe da estrutura do MR-MPS-SW

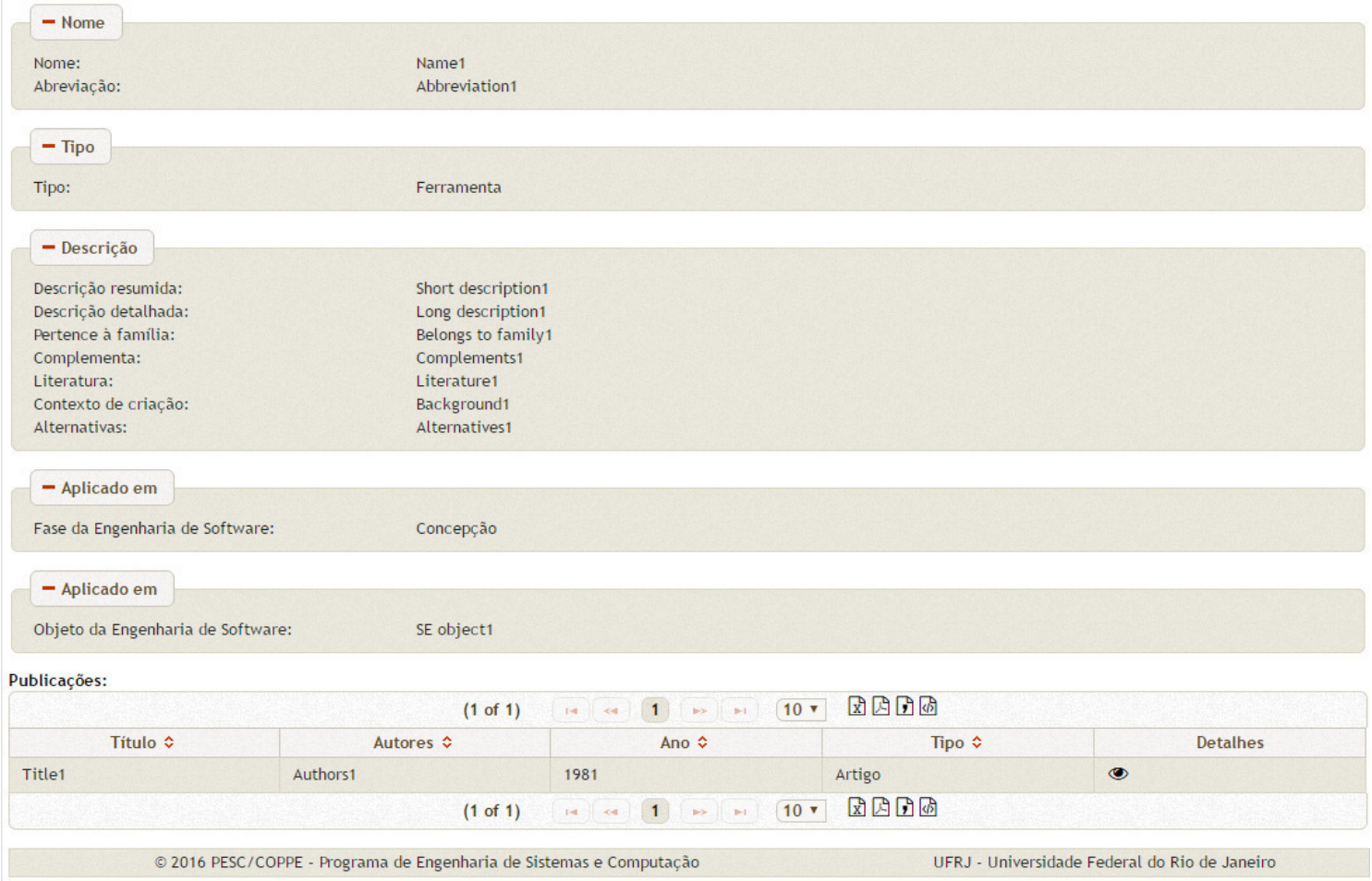

Figura 7 - Detalhes da tecnologia no MPS-BoK.

As informações apresentadas são provenientes da importação das informações de planilhas resultantes de execuções do MPS-BoK-P. Esta importação é realizada através de uma funcionalidade cujo acesso é restrito às pessoas responsáveis pela manutenção do MPS-BoK, através de usuário e senha. Esta funcionalidade permite a importação automática da planilha através de upload.

Tecnologias que possuem o mesmo nome agregam todas as publicações relacionadas. A ferramenta identifica um nome da tecnologia para realizar esta agregação automática como sendo o mesmo após a aplicação de técnicas de tratamento de texto. Uma bag of words [Harris 1981] foi construída retirando-se as stop words [Rajaraman and Ullman 2011] e comparando as palavras na ordem, sendo consideradas palavras iguais as que possuírem a distância Levenshtein menor ou igual a 2 [Levenshtein 1966]. 

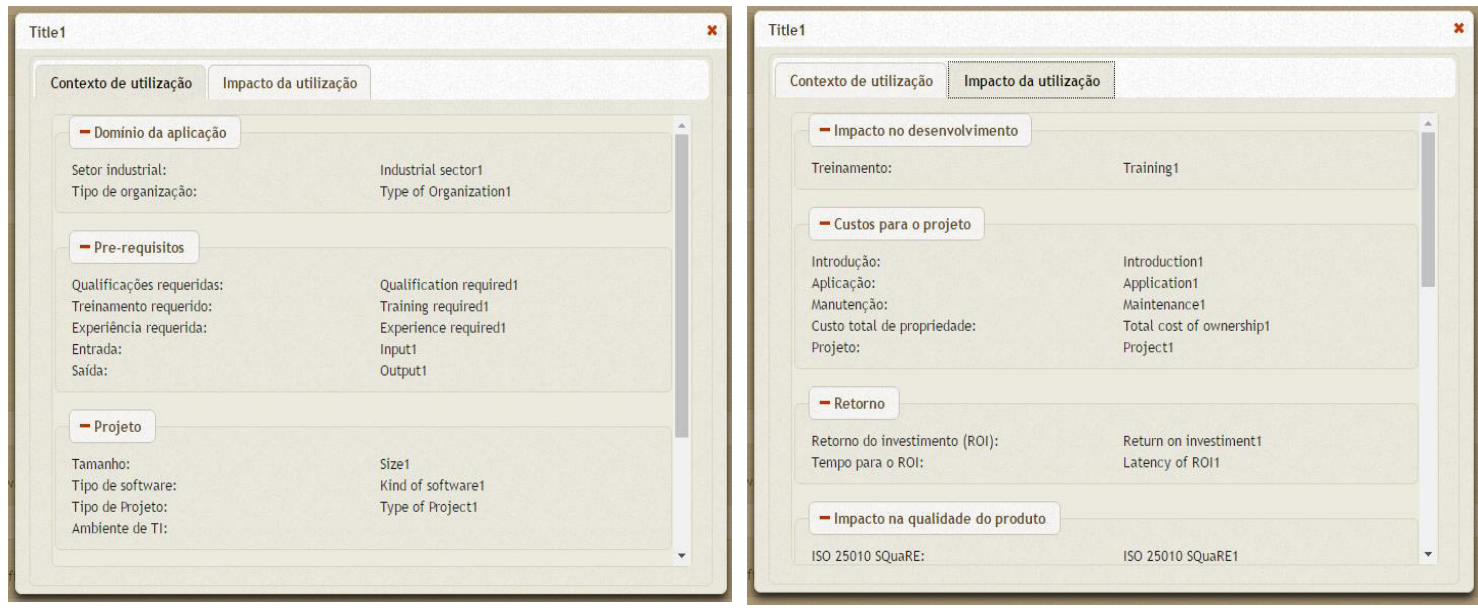

Figura 8 - Detalhes do contexto e impacto de utilização da tecnologia.

\section{Avaliação da infraestrutura proposta para o MPS-BoK}

A extração de conhecimento por pesquisadores para posterior adição ao MPS-BoK foi avaliada por ter sido considerada parte fundamental do MPS-BoK-P. Outra avaliação teve como foco a utilização da ferramenta de apoio ao MPS-BoK por profissionais envolvidos com melhoria de processos de software em organizações que desenvolvem software. Neste artigo um resumo destas avaliações é apresentado, cujos detalhes podem ser vistos em Lupo [2016].

Para estas avaliações foram definidos os objetivos de acordo com o "G" oal da abordagem Goal/Question/Metric (GQM) [Basili et al. 1994]. Esta abordagem apresenta uma estrutura para a definição de objetivos considerada adequada para este trabalho. Esta estrutura é definida como "Analisar o $<$ objeto de estudo $>$ com a finalidade de $<$ objetivo $>$ com respeito à $<$ foco da qualidade $>$ do ponto de vista de $<$ perspectiva $>$ no contexto de $<$ contexto $>$ ".

Além disso, foram formulados questionários baseados no TAM. O TAM (Technology Acceptance Model) [Davis 1989; Davis et al. 1989] foi utilizado por ser considerado adequado para medir a aceitação e a intenção de uso de novas tecnologias [Turner et al. 2010]. Assim, as perguntas foram definidas de acordo com o TAM e foram respondidas com a utilização de uma escala Likert [Likert 1932], suprimida a opção "neutro" (central) para evitar que neutralidades em um pequeno grupo de respondentes inviabilizem conclusões. Também foram incorporados mecanismos para que os participantes dessem sua opinião sobre cada um dos aspectos avaliados no TAM.

\subsection{Avaliação TAM da planilha de extração do conhecimento}

O objetivo desta avaliação foi: "Analisar a planilha de extração de informação com a finalidade de caracterizar com respeito à utilidade percebida, ao esforço da adoção e à intenção de adoção do ponto de vista do pesquisador no contexto da atividade Extração de dados do MPS-BoK-P a partir de artigos científicos".

Pela definição do perfil do usuário que executa esta atividade, de acordo com o MPS-BoK-P, os participantes devem ser pesquisadores com conhecimento do MRMPS-SW. Esta qualificação representa a caracterização necessária. Assim, tendo em vista a caracterização precisa, a técnica de seleção da amostra foi convenience sampling. 
Os participantes convidados foram todos doutores na área de Engenharia de Software e avaliadores do MR-MPS-SW.

Durante a operação, um artigo [Pinheiro et al. 2015] foi fornecido para a extração das informações para a planilha, o formulário foi preenchido e as respostas foram agregadas. É importante ressaltar que o objetivo da realização da extração foi que os participantes pudessem responder às questões de posse de uma experiência real, não caracterizando um experimento, mas uma experiência de uso para que pudessem opinar na avaliação. Ao todo 7 pesquisadores foram convidados a participar do estudo e 5 aceitaram.

Após a extração e o preenchimento da planilha, cada participante respondeu ao questionário posicionando-se em relação às perguntas e, a seu critério, fornecendo detalhamentos adicionais nos campos de observações. Os tempos de execução das atividades por cada um dos participantes, incluindo o tempo de extração das informações, foram $1 \mathrm{~h} 20 \mathrm{~min}, 1 \mathrm{~h} 21 \mathrm{~min}, 1 \mathrm{~h} 50 \mathrm{~min}, 2 \mathrm{~h} 15 \mathrm{~min}$ e $2 \mathrm{~h} 25 \mathrm{~min}$. Os resultados da avaliação em relação às dimensões do TAM (utilidade percebida, esforço de adoção e intenção de uso) estão sumarizados a seguir.

Utilidade Percebida. As respostas sobre a utilidade percebida se posicionaram de maneira equilibrada em todas as perguntas com a dificuldade de compreensão dos campos tendo sido consistentemente considerada nos comentários como um fator que contribuiu negativamente no resultado.

Esforço da Adoção. Quanto ao esforço de adoção, as respostas foram relativamente homogêneas tendendo levemente a resultados positivos, valendo ressaltar que houve diversos comentários relacionados à dificuldade de compreensão dos campos a serem preenchidos e de utilização da planilha devido à formatação e à validação, que precisam ser tratados como insumos para melhorias futuras.

Intenção de Uso. Apesar das respostas indicarem uma percepção majoritariamente negativa dos participantes em relação à intenção de uso, os comentários mostram que dois responderam de forma negativa por não possuírem familiaridade com o MPS-BoK e os outros dois citam que os fatores impeditivos são os problemas relacionados ao entendimento dos campos, formatação e restrições de edição impostas pela validação que influenciaram negativamente suas respostas. Estes problemas precisam ser mitigados através de melhorias na planilha e de um melhor esclarecimento quanto ao propósito de sua utilização.

\subsection{Avaliação TAM da ferramenta de apoio ao MPS-BoK}

O objetivo desta avaliação foi: "Analisar a ferramenta de apoio ao MPS-BoK com a finalidade de caracterizar com respeito à utilidade percebida, esforço da adoção e intenção de adoção do ponto de vista do profissional de melhoria de processos de software familiarizado com o MR-MPS-SW no contexto da utilização da ferramenta na execução de uma atividade relacionada à busca de conhecimento para melhoria de um processo de medição aderente ao MR-MPS-SW". Novamente um questionário foi construído a partir do TAM para capturar a percepção do praticante.

Tendo a necessidade de envolver profissionais de melhoria de processos familiarizados com o MR-MPS-SW, a amostra foi selecionada utilizando convenience sampling. A caracterização dos participantes foi realizada através de um questionário espe- 
cífico com a intenção de avaliar a experiência atuando com melhoria de processos e conhecimento do MR-MPS-SW.

Para a execução desta avaliação foi construído um cenário no qual uma organização fictícia precisa melhorar um processo de medição. A necessidade de melhoria do processo foi descrita e os participantes solicitados a executar uma atividade na forma de passos a serem seguidos na ferramenta visando chegar a um conhecimento que os apoie na tomada de decisão para o alcance da melhoria desejada. Novamente, o objetivo da realização da atividade foi que os participantes pudessem responder às questões familiarizados com a ferramenta e sua utilização, não caracterizando um experimento.

Com o propósito de alimentar o MPS-BoK com conhecimento sobre o processo Medição do MR-MPS-SW, o MPS-BoK-P foi executado pelo primeiro autor. Ao todo foram extraídas informações de 10 tecnologias a partir de 9 artigos associados com o processo Medição identificados entre os anos de 2003 a 2015 nos anais do Simpósio Brasileiro de Qualidade de Software (SBQS) e do Workshop Anual do MPS (WAMPS).

O formulário de caracterização e o do TAM foram preenchidos pelos participantes após a conclusão da tarefa de uso da ferramenta. Foram obtidas respostas de 4 profissionais que atuam na área de melhoria de processos de software de organizações diferentes, sendo duas grandes empresas (um banco e uma empresa do setor de energia) e duas médias (uma fábrica de testes e uma fábrica de software) e que possuem conhecimento do MR-MPS-SW (todos são ou já foram avaliadores do modelo). A caracterização dos participantes pode ser vista na Figura 9. É possível observar que se trata de profissionais extremamente experientes e realmente atuantes com melhoria de processos, representando uma amostra apropriada da população alvo do estudo. Os resultados em relação às dimensões do TAM se encontram sumarizados a seguir.

Utilidade Percebida. No geral as respostas sobre a utilidade percebida foram consideradas positivas, sendo frequentes os comentários sobre campos não preenchidos e questões relacionadas ao contexto específico de um dos participantes que podem ter contribuído negativamente.

Esforço da Adoção. Todos os participantes deram respostas positivas em todas as perguntas relacionadas ao esforço de adoção, com comentários também positivos.

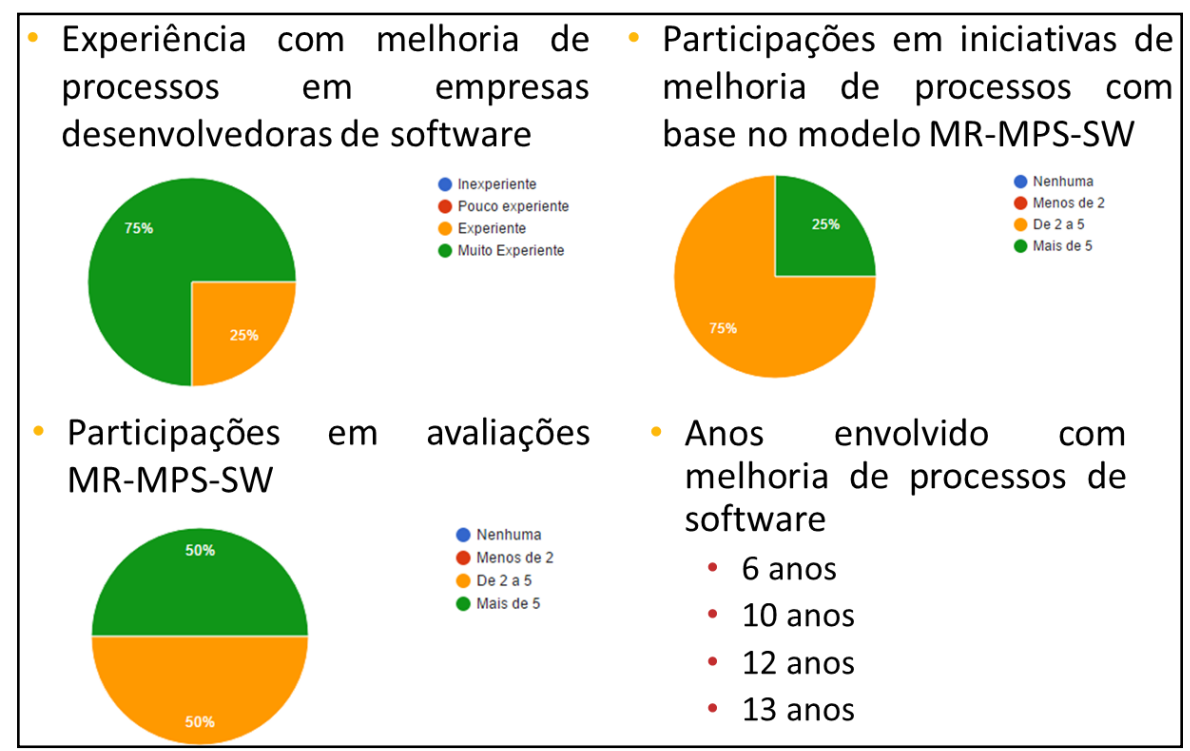




\section{Figura 9 - Caracterização dos Participantes.}

Intenção de Uso. A avaliação da utilidade da ferramenta foi uma das perguntas com mais dispersão nas respostas. A única avaliação negativa, no entanto, se deve ao participante $\mathrm{C}$, que justifica sua avaliação dizendo que no seu trabalho atual não há o costume de se realizar melhorias em processos com base em conhecimento externo à organização. Os outros participantes indicaram uma percepção positiva da utilidade da ferramenta de acesso ao MPS-BoK e forneceram sugestões sobre o tipo de conhecimento que a tornaria ainda mais útil e informações a serem apresentadas em associação com o conhecimento. Estas sugestões podem ser alvo de trabalhos futuros.

\section{Considerações Finais}

Neste artigo foi descrita uma infraestrutura para a organização de um BoK sobre tecnologias que podem ser utilizadas na melhoria de processos de software com base no MRMPS-SW, o MPS-BoK. A infraestrutura foi projetada para permitir a organização do MPS-BoK de maneira incremental e colaborativa através de um processo para atualizálo, o MPS-BoK-P, e de uma ferramenta que automatiza a organização do conhecimento extraído neste processo e o disponibiliza no MPS-BoK.

As principais contribuições referentes à concepção da infraestrutura apresentada neste trabalho são: (i) um processo simples que permite a atualização do conhecimento de maneira incremental, o MPS-BoK-P, definido através de uma especialização do SKE [Biffl and Kalinowski and Rabiser and et al. 2014] combinada com definições fornecidas por KUHRMANN et al. [Kuhrmann et al. 2015]; (ii) um modelo de domínio capaz de representar o MR-MPS-SW e informações associados aos seus elementos de modo a satisfazer as necessidades de profissionais (conforme definido em [Jedlitschka et al. 2014]); (iv) uma planilha para extração das informações das publicações de acordo com o modelo definido (ativo do processo MPS-BoK-P); e (v) uma ferramenta para organizar e disponibilizar conhecimento resultante de diferentes execuções deste processo, integrando informações sobre as tecnologias automaticamente e provendo mecanismos de busca textual utilizando recursos de operadores booleanos além de consulta através dos elementos do MR-MPS-SW.

No contexto das avaliações, o MPS-BoK-P foi executado pelo primeiro autor para preencher o MPS-BoK com conhecimento a respeito de tecnologias referentes ao processo de medição de software provenientes de artigos do SBQS e do WAMPS. Foram realizadas avaliações sobre elementos-chave da proposta através de questionários elaborados a partir do TAM direcionados ao público alvo. Estas avaliações fornecem indícios preliminares de viabilidade e elementos importantes para subsidiar trabalhos futuros. Os trabalhos futuros compreendem a realização dos ajustes e melhorias identificados nas avaliações para a disponibilização final do MPS-BoK, a generalização da solução para outros modelos de maturidade e novas avaliações.

É importante ressaltar que o interesse dos profissionais no MPS-BoK está associado aos conhecimentos incorporados pelos pesquisadores. Desta forma, o sucesso do BoK depende diretamente do engajamento da comunidade científica na contribuição de conteúdo através da execução do MPS-BoK-P.

\section{Referências}

Basili, V. R., Caldiera, G. and Rombach, H. D. (1994). The Goal Question Metric 
Approach. Wiley.

Biffl, S., Kalinowski, M., Ekaputra, F. J., Serral, E. E. and Winkler, D. (2014). Building Empirical Software Engineering Bodies of Knowledge with Systematic Knowledge Engineering. In International Conference on Software Engineering and Knowledge Engineering (SEKE).

Biffl, S., Kalinowski, M., Rabiser, R., Ekaputra, F. and Winkler, D. (29 dec 2014). Systematic Knowledge Engineering: Building Bodies of Knowledge from Published Research. International Journal of Software Engineering and Knowledge Engineering, v. 24, n. 10, p. 1533-1571.

Cerdeiral, C. T. (2014). Implantação de Inovações Tecnológicas e de Processo em Organizações de Software. Tese de Doutorado. UFRJ.

Chinn, P. L. and Kramer, M. K. (1995). Theory and nursing: a systematic approach. Mosby.

CMMI Product Team (2010). CMMI for Development, Version 1.3. Carnegie Mellon University. http://repository.cmu.edu/sei/287/.

Davis, F. D. (sep 1989). Perceived Usefulness, Perceived Ease of Use, and User Acceptance of Information Technology. MIS Quarterly, v. 13, n. 3, p. 319.

Davis, F. D., Bagozzi, R. P. and Warshaw, P. R. (aug 1989). User Acceptance of Computer Technology: A Comparison of Two Theoretical Models. Management Science, v. 35, n. 8, p. 982-1003.

Harris, Z. S. (1981). Distributional Structure. Dordrecht: Springer Netherlands.

Jedlitschka, A., Juristo, N. and Rombach, D. (11 dec 2014). Reporting experiments to satisfy professionals' information needs. Empirical Software Engineering, v. 19, n. 6, p. 1921-1955.

Kalinowski, M., Santos, G., Reinehr, S., et al. (2010). MPS . BR : Promovendo a Adoção de Boas Práticas de Engenharia de Software pela Indústria Brasileira. In XIII Congreso Iberoamericano en" Software Engineering"(CIBSE). http://www.softex.br/mpsbr/_guias/default.asp.

Kalinowski, M., Weber, K., Franco, N., et al. (2014). Results of 10 years of software process improvement in Brazil based on the MPS-SW model. Proceedings - 2014 9th International Conference on the Quality of Information and Communications Technology, QUATIC 2014, n. December 2003, p. 28-37.

Kitchenham, B. and Charters, S. (2007). Guidelines for performing Systematic Literature Reviews in Software Engineering. Engineering, v. 2, p. 1051.

Kuhrmann, M., Konopka, C., Nellemann, P., Diebold, P. and Münch, J. (aug 2015). Software process improvement: where is the evidence? Initial findings from a systematic mapping study. In Proceedings of the 2015 International Conference on Software and System Process - ICSSP 2015. . ACM Press. http://dl.acm.org/citation.cfm?doid=2785592.2785600.

Levenshtein, V. (1966). Binary Codes Capable of Correcting Deletions, Insertions and Reversals. Soviet Physics Doklady, v. 10, p. 707. 
Likert, R. (1932). A technique for the measurement of attitudes. Archives of Psychology, Lupo, P. P. (2016). MPS BOK - Infraestrutura para a Construção de um Corpo de Conhecimento em Melhoria de Processos de Software. Dissertação de Mestrado. UFRJ.

Montoni, M. A. (2010). Uma investigação sobre os fatores críticos de sucesso em iniciativas de melhorias de processos de software. Tese de Doutorado. UFRJ.

Oliver, G. R. (2012). Foundations of the Assumed Business Operations and Strategy Body of Knowledge (BOSBOK): An Outline of Shareable Knowledge. Darlington Press.

Ören, T. I. (2005). Toward the Body of Knowledge of Modeling and Simulation. In Interservice/Industry Training, Simulation, and Education Conference (I/ITSEC) 2005. . http://www.site.uottawa.ca/ oren/pubs-pres/2005/pub-0513-MSBOK-IITSEC.pdf.

Pinheiro, G., Souza, P. J. S. De, Ronaldo, S. and Oliveira, B. (2015). SpiderMsControl : Uma Ferramenta para Apoio ao Processo de Medição usando a Abordagem GQIM. In Workshop Anual do MPS.

Rajaraman, A. and Ullman, J. D. (2011). Data Mining. Cambridge: Cambridge University Press.

Richardson, W. S., Wilson, M. C., Nishikawa, J. and Hayward, R. S. (jan 1995). The well-built clinical question: a key to evidence-based decisions. ACP journal club, v. 123, n. 3, p. A12-3.

Santos, G., Kalinowski, M., Rocha, A. R., et al. (2012). MPS.BR program and MPS model: Main results, benefits and beneficiaries of software process improvement in Brazil. Proceedings - 2012 8th International Conference on the Quality of Information and Communications Technology, QUATIC 2012, p. 137-142.

Shull, F., Carver, J., Travassos, G. H., et al. (2003). Replicated Studies: Building a Body of Knowledge about Software Reading Techniques. Lecture Notes on Empirical Software Engineering. WORLD SCIENTIFIC. p. 39-84.

Softex (2016). Guia Geral MPS de Software. Associação para Promoção da Excelência do Software Brasileiro - SOFTEX.

Travassos, G. H. and Kalinowski, M. (2014). iMPS 2013: Evidências Sobre o Desempenho das Empresas que Adotaram o Modelo MPS-SW. Campinas, SP: Softex Associação para Promoção da Excelência do Software Brasileiro.

Turner, M., Kitchenham, B., Brereton, P., Charters, S. and Budgen, D. (may 2010). Does the technology acceptance model predict actual use? A systematic literature review. Information and Software Technology, v. 52, n. 5, p. 463-479. 